\title{
COUCHES EXTERIEURES ET STRUCTURE INTERNE DES ÉTOILES
}

\author{
Invited Discourse B given on August 25, 1967 in the Lucerna Hall \\ P. LEDOUX \\ (Institut d'Astrophysique, Université de Liège, Belgique)
}

Monsieur le Président, Mesdames, Mesdemoiselles, Messieurs,

C'est un honneur redoutable que d'être invité à prendre la parole devant cette imposante assemblée, d'autant plus redoutable dans ce cas, qu'en l'acceptant j'ai assumé deux tâches parallèles: d'une part, celle de renforcer quelque peu, et espéronsle honorablement, la présence d'une des deux langues officielles de l'Union et, d'autre part, celle de vous présenter un problème qui relève certes du domaine de mes intèrêts mais qui auraît pu trouver parmi vous des porte-paroles sans aucun doute plus attitrés et plus compétents.

Le problème des interactions.entre la structure interne des étoiles et les propriétés de leurs couches extérieures présente une grande variété d'aspects dont certains, par leur complexité, peuvent échapper longtemps encore à tout effort d'élucidation. C'est d'autre part un problème fondamental car, à part des renseignements précieux sur la masse et la luminosité totales des étoiles et, dans quelques cas assez rares, sur leurs rayons et sur leur condensation centrale et, peut-être à l'avenir grâce aux neutrinos, quelques renseignements directs sur les réactions nucléaires qui se déroulent dans le soleil, toutes nos connaissances sur la constitution interne des étoiles dérivent, en fin de compte, de la possibilité d'extrapoler, vers l'intérieur, les conditions directement observées dans les couches atmosphériques les plus extérieures dont la masse ne représente cependant qu'une fraction infime de la masse totale.

Dans le passé, cette dernière circonstance a souvent été invoquée pour justifier l'usage dans la théorie de la structure interne des étoiles de conditions aux limites fort sommaires à la surface. Les développements ultérieurs que nous nous proposons de passer en revue ici nous incite à présent à plus de prudence, mais on peut néanmoins se demander si toutes les propriétés de ces couches ou certains détails de leur structure sont réellement significatifs pour l'intérieur de l'étoile. L'agitation générale à la surface $\mathrm{du}$ soleil, la granulation, les ondes plus régulières qu'on y discerne à plus grande échelle, les champs magnétiques irréguliers et leurs variations au cours du temps, la rotation différentielle, la présence d'une chromosphère et d'une couronne, le vent solaire lui-même sont-ils des phénomènes d'origine purement superficielle sans consé-

Perek (ed.), Highlights of Astronomy, 12-32. (C) I.A.U. 
quences pour la constitution des couches internes? Sont-ils au contraire le reflet de certaines conditions réalisées en profondeur? Les mêmes questions peuvent se poser à propos des champs magnétiques stellaires, de certains types de variabilités erratiques ou régulières, de la distribution des vitesses de rotation ou, encore, à propos des anomalies d'abondances ou même des abondances moyennes observées dans les atmosphères. Et dans les circonstances de l'astrophysique, il est bien difficile d'y répondre sinon par l'élaboration patiente de toutes les possibilités théoriques que la présence de ces facteurs suggère, l'élimination progressive d'un certain nombre d'entre elles et la mise en évidence de l'importance de quelques autres soit par des déductions logiques basées sur une bonne connaissance de la physique stellaire soit par la découverte de corrélations entre la présence et l'intensité de ces facteurs et d'autres données significatives telles que l'âge des étoiles ou leur appartenance à différents systèmes ou sous-systèmes stellaires. Mais d'ailleurs dans la plupart des cas, nous en sommes réduits à une information fort limitée concernant essentiellement la température et la gravité dans les couches superficielles et la luminosité totale de l'étoile. Historiquement, le problème de la structure interne a été formulé tout naturellement en termes des trois paramètres correspondants, la masse totale $M$, le rayon $R$, et la luminosité $L$.

Même ainsi, la nature du problème doit nous inciter à quelque prudence. En effet, supposons que la composition chimique est connue soit qu'elle soit supposée uniforme et identique à celle de l'atmosphère soit que des calculs préliminaires liés à l'étude de l'évolution stellaire aient fourni sa variation avec la profondeur. Supposons également que nous disposons de toutes les lois physiques appropriées: équations d'état très générales, opacité, taux de génération d'énergie ainsi que de critères locaux permettant de juger de l'importance relative des différents modes de transport d'énergie. Dans ce cas, la construction d'un modèle stellaire instantané en équilibre hydrostatique ou quasi-statique, pour une masse donnée $M$, revient à chercher la (ou éventuellement les) solution d'un système différentiel du quatrième ordre dépendant de $R$ et $L$ comme paramètres et qui satisfait (ou satisfont) à deux conditions au centre et à deux conditions à la surface. Comme divers auteurs l'ont fait remarquer (1), il s'agit là, en réalité, d'un problème aux limites fortement non linéaire et, à ma connaissance, la question de l'existence et de l'unicité de la solution n'a pas reçu jusqu'ici de réponse satisfaisante d'un point de vue mathématique strict. Cependant, l'expérience considérable acquise dans le domaine des modèles stellaires, grâce notamment aux grands ordinateurs électroniques, montre qu'en pratique la solution existe et qu'elle est presque toujours unique sauf dans de rares cas comme celui, p. ex., des modèles possédant un noyau isotherme ou quelque autre type de noyau très condensé de masse relative donnée. Mais dans ces cas, sans doute, une seule des solutions possibles est-elle réellement stable et peut-être même le calcul continu de l'évolution stellaire nous conduit-il automatiquement et univoquement à celle-ci bien qu'à l'occasion, une telle situation puisse soulever des problèmes délicats là notamment où deux telles séries stable et instable se rejoignent ou se croisent. 
Une autre caractéristique de ces problèmes aux limites est la sensitivité souvent considérable des solutions aux conditions exactes imposées aux extrémités de l'intervalle d'intégration. Ceci est déjà apparent dans les modèles les plus simples définis par une relation polytropique $P=K \rho^{\gamma}$ ou, paramétriquement, $P=P_{c} \theta^{n+1}, \rho=\rho_{c} \theta^{n}$. Dans ces modèles, la surface est déterminée par la condition $\theta=0$ (c.-à-d., physiquement $P=\rho=T=0$ ) pour une valeur de la variable radiale d'Emden disons $\xi_{s}$ qui peut être prise égale à l'unité. Les solutions construites à partir de ce point sont de différents types: pour une valeur bien déterminée $(\mathrm{d} \theta / \mathrm{d} \xi)_{s, E}$ de la dérivée en $\xi_{s}=1$, la solution, dite d'Emden, croît uniformément pour atteindre le centre $\xi=0$ avec une valeur finie et un coefficient angulaire nul; pour des valeurs initiales $\mathrm{de}(\mathrm{d} \theta / \mathrm{d} \xi)_{s}$ plus petites en valeurs absolues, les solutions tendent vers l'infini au centre et sont dites solutions $M$ ou 'centrally condensed', tandis que pour des valeurs initiales plus grandes que $(\mathrm{d} \theta / \mathrm{d} \xi)_{s, E}$ les solutions tendent à s'annuler de nouveau avant d'atteindre le centre et sont dites solutions $\mathrm{F}$ ou du type 'collapsed'.

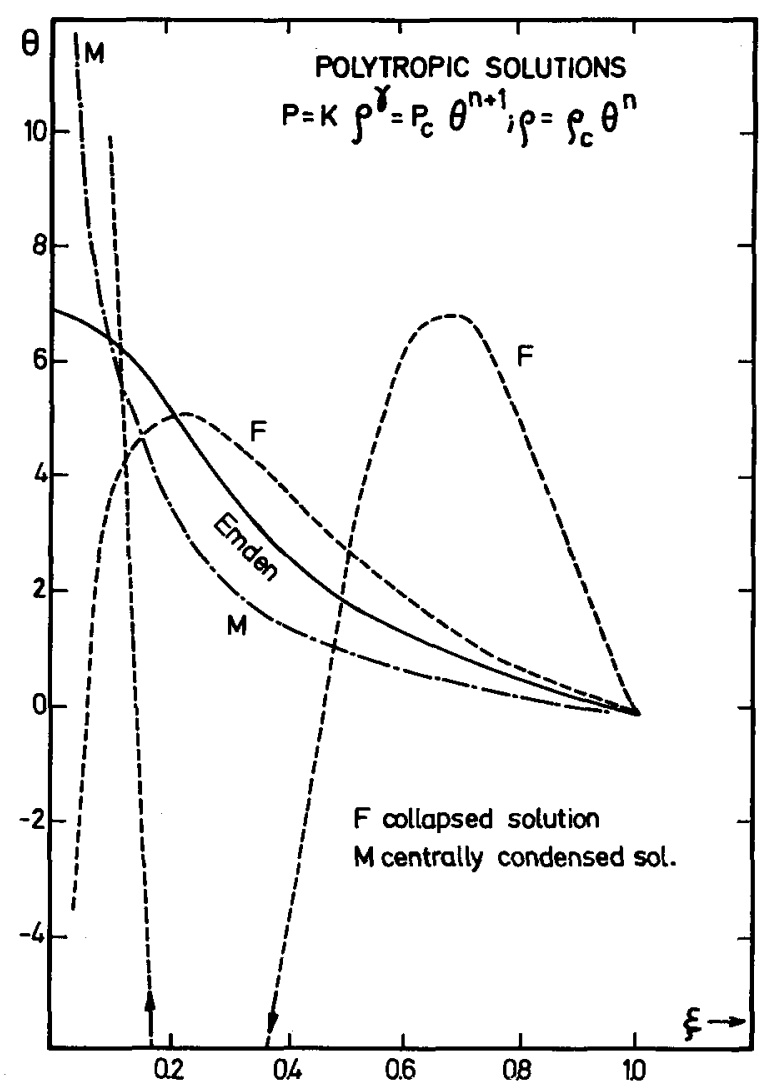

Fig. 1. Illustration des différents types de solutions polytropiques. 
L'existence des solutions des types $\mathrm{F}$ et $\mathrm{M}$ était déjà connue d'Emden mais leur discussion doit beaucoup (2) à l'intérêt que Milne (3) y prit. A cette époque déjà lointaine où bien des données physiques faisaient défaut, la considération de ces solutions et particulièrement des solutions 'centrally condensed' ainsi que de conditions à la surface plus précises tenant compte de la température finie qui y règne lui paraissait justifier une remise en question générale de vues courantes sur la structure interne des étoiles. Bien que ce point de vue extrême se soit vite révélé injustifié (4), il n'en reste pas moins que l'assertion de Milne concernant l'impossibilité de déterminer univoquement la composition chimique profonde à partir de $M, L$ et $R$ ou des relations qui les lient a reçu de nos jours une confirmation au moins partielle. De même, certains résultats formels issus des recherches qu'il incita ont pavé la voie à des developpements importants.

À ce dernier point de vue, nous sommes particulièrement intéressés ici par une des conditions de jonction dans les configurations composites, à savoir qu'une solution extérieure d'indice polytropique plus petit que celui du noyau central ne peut être réunie à celui-ci que si elle est du type 'centrally condensed'. De plus, c'est la valeur de la constante $K$ dans la relation $P=K \rho^{\gamma}$ qui permet de distinguer entre elles les solutions extérieures de ce type. Or la valeur de $K$ sera déterminée par les valeurs de $P$ et $\rho$ obtenues par intégration à partir de la mince frange atmosphérique en équilibre radiatif au travers d'une zone de transition sous-jacente plus ou moins profonde à la base de laquelle la solution polytropique ou pseudo-polytropique envisagée devient valable. Dans ces circonstances, la structure interne profonde peut être fortement affectée par les conditions dans les couches extérieures et en particulier par l'ionisation de l'hydrogène.

En fait, dès 1930, Unsöld (5) fit remarquer que cette ionisation peut réduire suffisamment les rapports généralisés $\Gamma$ des chaleurs spécifiques pour créer une instabilité convective violente dans les couches subphotosphériques des étoiles de températures effectives pas trop élevées, disons inférieures à $10000^{\circ}$. Dans le cas du soleil, il trouva que la convection ne pouvait manquer de se développer à partir d'une profondeur optique relativement faible de l'ordre de $\tau=3$ et il n'hésita pas à y rattacher l'origine de la granulation observée aux niveaux photosphériques.

$\mathrm{Au}$ début, ce fut sans doute cette dernière possibilité qui retint l'attention et qui consacra l'intérêt de la découverte de Unsöld. Dans la suite, l'importance de cette zone ne fit que croître au fur et à mesure que la prédominance de l'hydrogène dans la composition chimique des étoiles s'affirmait et que le calcul précis de l'opacité aux basses températures $\left(T<10^{4} \mathrm{~K}\right)$ due à l'hydrogène et son ion négatif (6) révélait que son accroissement rapide avec la température hâtait encore l'apparition à faible profondeur de l'instabilité convective. D'autre part, les travaux de Biermann (7) en particulier attirèrent bientôt l'attention sur le fait que la zone de convection de l'hydrogène pouvait s'étendre bien plus profondément qu'on ne l'avait prévu initialement. En effet, les premières discussions reposaient sur la considération d'enveloppes 
en équilibre purement radiatif dans lesquelles l'instabilité convective due à l'ionisation de l'hydrogène était limitée à une zone relativement étroite et, malgré la violence de cette instabilité, on admettait généralement en première approximation que la convection ne s'étendrait guère qu'à cette zone. Mais en réalité, la convection en s'établissant réduit le gradient de température, là particulièrement où les $\Gamma$ sont petits, ce qui tend déjà à étendre vers le bas la région de l'ionisation critique. De plus, les températures relativement basses qu'elle associe ainsi à des pressions ou densités données, même bien au-delà de la zone d'ionisation de l'hydrogène, réduisent très fortement la contribution du flux radiatif au transport total d'énergie si bien que la convection persiste jusqu'à des profondeurs considérables (8). En réalité, dans ces régions où les $\Gamma$ reprennent rapidement leurs valeurs normales, la convection est si efficace que la distribution des conditions physiques y est pratiquement isentropique et correspond, dans le cas où la pression de radiation est négligeable, à une des solutions polytropiques $n=\frac{3}{2}, P=K \rho^{5 / 3}=Q T^{5 / 2}$.

Malheureusement, il n'en est pas de même dans la partie supérieure de la zone convective de l'hydrogène. Dans cette région, l'efficacité de la convection est beaucoup plus faible par suite de la capacité calorifique réduite, de la transparence relative de la matière et de la limite supérieure relativement basse que la vitesse du son y impose aux vitesses de convection. Les gradients de température et de densité qui s'y établissent à l'équilibre dépendent des valeurs relatives du flux radiatif et du flux convectif et ce dernier devrait donc pouvoir être évalué, comme le premier, directement en termes des conditions physiques. Malheureusement, nous n'avons guère à notre disposition qu'une théorie de la convection turbulente dans laquelle le libre parcours moyen $l_{t}$ reste un paramètre relativement arbitraire. Certes, il peut être associé à quelque sousmultiple de l'épaisseur totale de la zone convective ou à la hauteur d'échelle des variations de la pression ou de la densité. Mais il dépend ainsi des grandeurs mêmes qu'on se propose de déterminer et l'utilisation d'un processus d'itération ne garantit même pas, dans tous les cas, des résultats exempts de toute contradiction interne. Afin de pallier à ces difficultés, différentes suggestions ont été avancées récemment tendant, p. ex., à lier le libre parcours moyen $l_{t}$ à une hauteur d'échelle moyenne plutôt qu'à sa valeur locale (9) ou même à se passer de l'usage, au moins explicite, de cette notion (10). Mais, dans les deux cas, les avantages réels paraissent plutôt minimes. D'autre part, il est peut-être un peu surprenant que, dans les développements en série des variables d'état par lesquels $l_{t}$ s'introduit dans la théorie, seuls les termes linéaires aient été retenus jusqu'ici alors qu'en tenant compte au moins des termes en $l_{t}^{2}$ on pourrait s'attendre à améliorer les cas les plus difficiles où, justement, les conditions varient très rapidement avec la profondeur.

De plus, les effets de la dissipation par conduction radiative, par conduction et friction turbulente aux échelles intermédiaires et par viscosité à l'échelle la plus petite compliquent encore le problème. Ceci d'ailleurs soulève la question du spectre de la convection turbulente et de l'utilisation des théories semi-heuristiques appropriés, en 
combinaison, peut-être, avec les résultats de l'étude des perturbations linéaires nonradiales de la couche (11). Cette analyse linéaire étendue aux facteurs non-conservatifs $\mathrm{du}$ problème pourrait peut-être fournir les dimensions et le temps de vie des perturbations les plus favorisées (12) fixant ainsi l'ordre de grandeur de $l_{t}$ dans la théorie élémentaire ou permettant, dans les théories plus raffinées, une normalisation nontotalement arbitraire du spectre.

En tout cas, l'insuffisance de nos théories actuelles de la convection est un handicap sérieux dans ce problème.

Dans ce qui précède, suivant en cela le développement historique de la question, nous avons mis l'accent sur les effets de l'ionisation de l'hydrogène mais il est clair que l'ionisation d'un autre élément abondant comme l'hélium peut entraîner également des effets importants qui peuvent d'ailleurs se manifester dans des classes d'étoiles distinctes et différer quelque peu p. ex. pour les étoiles de population I et II.

Peut-être n'avons-nous pas non plus assez insisté sur l'importance des tables détaillées d'opacité, pour les températures et densités intermédiaires, mises à notre disposition par quelques chercheurs (13) passés maîtres en physique atomique et disposant de puissants ordinateurs électroniques. Sans leurs résultats, bien des progrès substantiels dans la physique difficile des couches de transition entre l'atmosphère et l'intérieur stellaire proprement dit n'auraient pas été possibles.

Enfin, une théorie satisfaisante des couches atmosphériques en équilibre radiatif est également nécessaire afin de nous fournir les conditions rêgnant au dessus de la couche d'ionisation de l'hydrogène. Ceci requiert au moins une bonne connaissance de l'opacité dans les couches très extérieures ce qui pose parfois des problèmes bien difficiles surtout aux très basses températures. Mais il est d'ailleurs d'autres effets (14) qui peuvent être importants dans certains cas et qui restent bien difficiles à évaluer: effet de serre, anisotropie de la radiation, courbure des atmosphères étendues, convection pénétrative, modification des conditions à la base de l'atmosphère qui, strictement, rend caduques (15) les solutions radiatives pour atmosphères semi-infinies généralement utilisées.

Comme d'habitude, dans les problèmes complexes de ce genre, les progrès sont issus d'attaques incomplètes certes mais efficaces comme celle de Vitense qui, dès 1953 (16) permit, dans le cas du soleil, d'encadrer la solution dans des limites raisonnables. Améliorée en 1958 (17), cette méthode a servi de modèle à presque toutes les investigations subséquentes. De plus, c'est en grande partie son emploi critique et ses généralisations qui ont dégagé peu à peu les différents aspects du problème, y compris ceux qui, jusqu'à présent, restent bien difficiles ou impossibles à traiter.

Malgré ces imperfections et l'indétermination qui pèse sur les solutions du fait de l'arbitraire qui subsiste dans le choix du libre parcours moyen $l_{t}$, une réelle moisson de résultats importants et d'implications intéressantes a déjà été récoltée, même en se bornant à la séquence principale. Si nous passons aux étoiles plus chaudes que le soleil, la zone d'ionisation de l'hydrogène se déplace vers la surface en s'amincissant 
et la convection qui en résulte devient de moins en moins efficace, la radiation devant assurer le transport d'une fraction de plus en plus grande de l'énergie. Ses effets sur la structure interne profonde s'atténuent donc progressivement pour disparaître pratiquement au-dessus d'une température effective de l'ordre de 8000 à $10000^{\circ} \mathrm{K}$ dans les dernières classes spectrales de type $\mathrm{A}$. Le rôle des ionisations de l'hélium dépend naturellement de l'abondance adoptée pour cet élément et, dans la population de type I, elles peuvent renforcer quelque peu les effets de l'ionisation de l'hydrogène mais leur influence semble cependant s'atténuer très rapidement dès que la température effective approche une dizaine de milliers de degrés $(\mathbf{1 8})$.

$\mathrm{Si}$, comme il paraît vraisemblable, une partie de l'activité solaire et la présence d'une chromosphère et d'une couronne sont liées étroitement à l'existence de la zone convective de l'hydrogène, on peut prévoir que des phénomènes du même genre se rencontreront sans doute jusque dans les premiers types spectraux F. Schatzman (19)

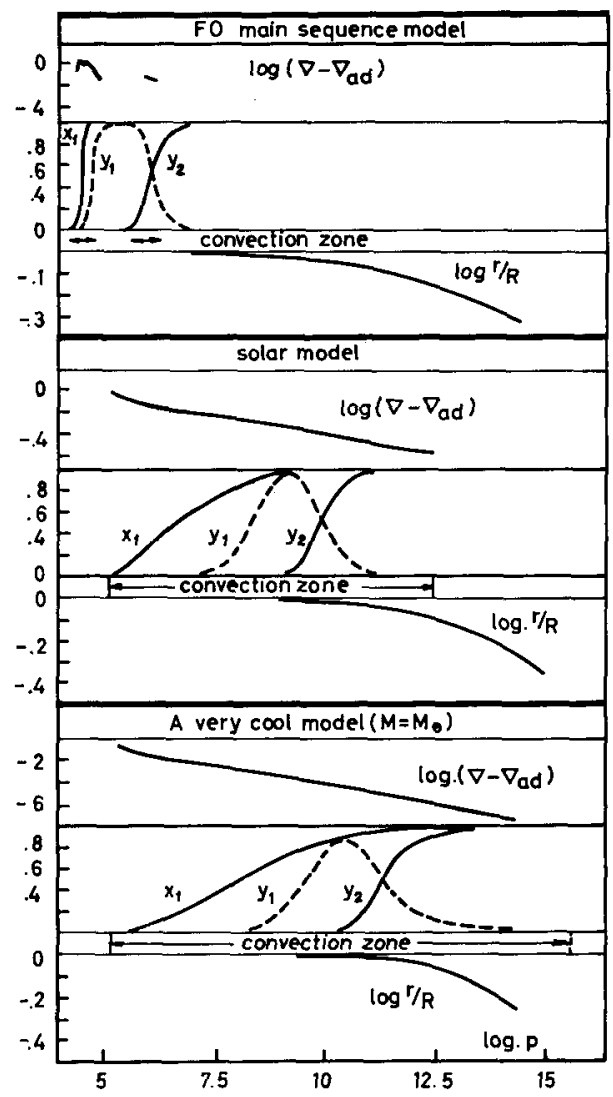

FIG. 2. Importance relative des zones de convection de l'hydrogène et de l'hélium pour différents modèles (tirée de Baker et Temesvary,(18)). 
considère d'ailleurs que, dans les classes spectrales plus froides, cette activité en présence de champs magnétiques peut conduire, au cours des dernières phases de la contraction gravifique, à une dissipation de moment cinétique capable d'expliquer la variation rapide de la vitesse de rotation qui se produit justement dans cette classe F. Peut-être, les nouvelles possibilités d'observation dans l'UV et dans le domaine des rayons $\mathrm{X}$ ouvertes par l'avènement des satellites artificiels pourront-elles un jour fournir des données décisives sur cette question?

D'autre part, si nous descendons le long de la séquence principale vers les étoiles moins chaudes que le soleil, l'influence de la zone d'ionisation de l'hydrogène, renforcée dans les couches très extérieures par les effets d'opacité et par la dissociation de la molécule $\mathrm{H}_{2}$, s'accentue et la convection tend à s'étendre de plus en plus profondément dans l'étoile. En même temps, son efficacité s'accroît dans les couches extérieures et tend à y établir des conditions isentropiques pratiquement jusqu'au point, bien au-dessus de la zone d'ionisation de l'hydrogène, où le critère de Schwarzschild est violé. Ainsi la région difficile à gradient superadiabatique qui compliquait fortement le problème dans les étoiles plus massives tend à s'évanouir ici. Dans ce cas, une simple zone à entropie constante peut servir à joindre, au travers des zones d'ionisation de l'hydrogène et de l'hélium, l'atmosphère radiative à la région convective profonde qui, pour ces faibles masses, peut être représentée par une solution polytropique $P=K \rho^{5 / 3}$ ou $P=Q T^{5 / 2}$ où $K$ et $Q$ ou encore $E^{*}$ reliée à $Q$ par

$$
E=4 \pi G^{3 / 2}\left(\frac{\mu}{R}\right)^{5 / 2} R^{3 / 2} M^{1 / 2} Q
$$

sont fixées par les valeurs que prennent les variables d'état là où l'ionisation de $\mathrm{H}$ et He devient complète.

Comme il s'agit de réunir éventuellement ces enveloppes convectives à un noyau radiatif dont l'index polytropique effectif sera certainement plus grand que $\frac{3}{2}$, seules, comme nous l'avons rappelé plus haut, des solutions polytropiques d'indice $n=\frac{3}{2} \mathrm{du}$ type 'centrally condensed' peuvent convenir pour représenter ces enveloppes. Elles correspondent à des valeurs de $E$ inférieures à la valeur $E=45.5$ qui caractérise la solution d'Emden. Elles doivent être réunies aux solutions radiatives issues du centre au point où, le long de ces dernières, l'index polytropique effectif devient égal à $\frac{3}{2}$. Sur la Figure 3, utilisée par Osterbrock (20) pour construire des modèles des étoiles de faible masse Castor $\mathrm{C}$ et $\alpha$ Cen $\mathrm{B}\left(0.65 \leqslant M / M_{\odot}<1\right)$, le lieu de ces points est indiqué et la jonction avec la solution 'centrally condensed' fixée par la valeur de $E$ se fait facilement par interpolation. Osterbrock établit ainsi que, pour les étoiles

* E est lié au paramètre classique

$$
\omega_{n}=-\xi_{s}^{\frac{n+1}{n-1}}\left(\frac{\mathrm{d} \theta_{n}}{\mathrm{~d} \xi}\right)_{s}
$$

défini par Chandrasekhar (I.S.S.S., Chap. IV, Eqs. 73, 337, 477) par la relation

$$
E=\left(\omega_{3 / 2}\right)^{1 / 2}\left(\frac{5}{2}\right)^{3 / 2} \text { si } n=\frac{3}{2} \text {. }
$$




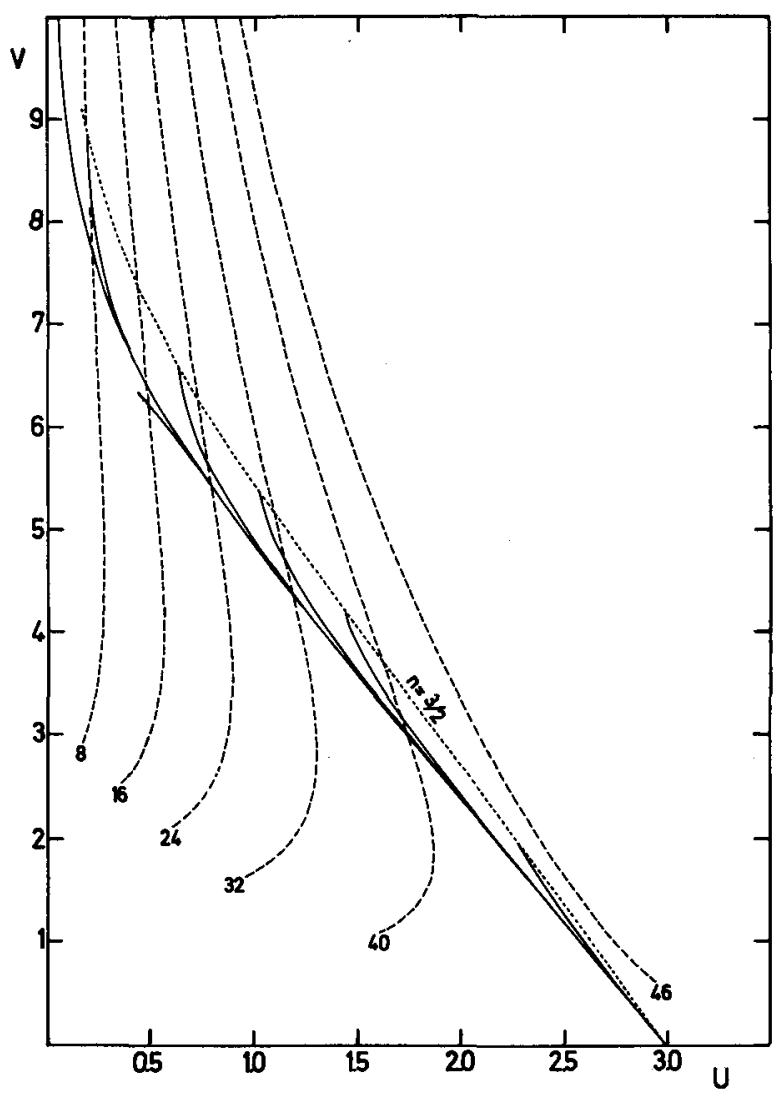

FIG. 3. Figure empruntée à l'article d'Osterbrock (20), et illustrant le problème de la jonction entre enveloppes convectives (courbes en trait interrompu correspondant à diverses valeurs de E) et noyaux radiatifs (courbes en trait plein). La courbe pointillée représente le lieu des points où le long des solutions radiatives $n$ devient égal à $\frac{3}{2}$.

considérées, l'enveloppe convective s'étend très profondément couvrant, pour $M=0 \cdot 65 M_{\odot}$, des fractions du rayon et de la masse égales respectivement à 0.33 et $0 \cdot 11$.

Ces résultats furent étendus successivement à des masses de plus en plus faibles par Limber (21), qui prend en considération les effets de la dégénérescence partielle des électrons et par Hayashi et Nakano (22), qui, en plus, tiennent compte de la molécule $\mathrm{H}_{2}$ et de sa dissociation. Ces études révélèrent que la convection s'étendait effectivement jusqu'au centre dans les masses plus petites ou égales à $0 \cdot 27 M_{\odot}$, conclusion qui fut confirmée récemment par une étude plus détaillée encore due à Gabriel (23). De plus, ce dernier travail montre que les modèles correspondants sont vibrationnellement instables sous l'action des réactions de la chaine $p$-p et des effets de la convection dans les zones extérieures d'ionisation, instabilité qui doit d'ailleurs s'étendre quelque peu au-dessus de la masse limite $0 \cdot 27 M_{\odot}$. 
Bien que cette instabilité ne soit pas très violente, elle devrait pouvoir se manifester, le temps d'amplification de l'ordre de $10^{8}$ années restant court vis-à-vis de la durée de vie de $10^{11}$ à $10^{12}$ années de ces étoiles. Devons-nous dès lors, nous attendre à y découvrir quelque forme de variabilité très rapide avec des périodes de l'ordre d'une vingtaine de minutes? Ou avons-nous laissé échapper quelque facteur capable de rétablir la stabilité au prix sans doute du rejet des modèles?

Quoiqu'il en soit, comme nous venons de le rappeler, l'influence des couches extérieures s'est révélée capitale pour l'interprétation des étoiles de la séquence principale des types les plus froids au type F. Mais, c'est sans doute dans l'étude de l'évolution stellaire que l'importance des conditions superficielles et des zones de transition extérieures a reçu la consécration la plus spectaculaire.

Dès que les principales réactions nucléaires d'intérêt stellaire eurent été isolées par Bethe et Von Weiszäcker en 1939, l'interprétation des étoiles de la séquence principale parût immédiatement assurée au moins en première approximation. Par contre, la structure des étoiles géantes et la signification des branches correspondantes dans les diagrammes de Hertzsprung-Russell des amas restaient mystérieuses (24). Un pas décisif fut accompli par Sandage et Schwarzschild (25) quand ils établirent l'existence de modèles à noyau d'helium pseudo-isotherme en contraction entouré d'une enveloppe en expansion permettant d'assurer la continuité de l'évolution au-delà du modèle critique de Schönberg-Chandrasekhar. Ce résultat fut d'ailleurs bientôt étendu (26) aux masses assez faibles pour que le gaz d'électron dégénère partiellement dans les régions centrales. Dans tous les cas, le chemin évolutif dans le diagramme de Hertzsprung-Russell tourne brusquement vers la droite par suite de l'accroissement rapide du rayon global quand la masse du noyau d'hélium atteint quelque $10 \%$ de la masse totale. Le coude caractéristique des amas anciens trouve ainsi une interprétation immédiate et sa position permet une estimation directe de leur âge. Mais l'évolution ultérieure sur la base de mêmes modèles à enveloppes purement radiatives dans lesquelles $T$ s'annule à la surface révélait bientôt une nouvelle difficulté. En effet, le point représentatif du modèle continuait à s'écarter vers la droite presque horizontalement au lieu de remonter vers le coin supérieur droit du diagramme après un court palier comme les données d'observation l'exigeaient.

En 1955, Hoyle et Schwarzschild (27) confrontés par cette difficulté remarquèrent que, quelque part le long de cette évolution, la température effective devient si basse que des conditions aux limites réalistes avec une température de surface finie requièrent, comme dans les modèles d'Osterbrock, l'existence d'une zone convective extérieure qui devient rapidement importante. En tenant compte de ceci, ils purent montrer que, pour des valeurs acceptables de la constante $E$, le chemin d'évolution se redresse vers le haut en accord raisonnable avec la branche observée des géantes rouges. Les nombreuses investigations ultérieures (28) adaptées aux divers perfectionnements auxquels nous avons déjà fait allusion confirmèrent entièrement ces résultats bien que l'indétermination qui pèse toujours sur le libre parcours moyen turbulent entraîne 
inévitablement une certaine marge d'incertitude sur la position de la branche théorique (voir (14)).

À l'autre extrémité de l'évolution stellaire, les travaux de Hayashi allaient bientôt dégager une autre conséquence fort inattendue et tout aussi importante puisqu'elle allait révolutionner nos vues sur les phases de contraction gravifique précédant l'arrivée sur la séquence principale. Au cours d'une investigation sur l'interprétation de la branche des géantes rouges (29), il remarque que, pour les configurations où la pression de radiation est négligeable, il ne peut pas exister de modèles en équilibre pour une valeur du paramètre $E$ supérieure à la valeur 45.5 associée à la solution d'Emden ce qui n'est rien d'autre que la proposition déjà rappelée qui limite les solutions acceptables pour l'enveloppe convective au type 'centrally condensed'. Comme la discussion n'est guère affectée qualitativement par la pression de radiation, nous continuerons ici à l'ignorer. Ainsi, pour une masse et une composition chimique données, on peut, par une théorie appropriée des couches photosphériques et de la zone de transition, exprimer le rapport $\left(P / T^{5 / 2}\right)=Q$ à la limite inférieure de cette region, là où commence la zone convective polytropique, en termes de la luminosité $L$ et de la température effective $T_{\mathbf{c}}$. Réintroduisant cette expression de $Q$ dans la définition de $E$ qui prend sa valeur critique $E=45 \cdot 5$, on obtient une équation qui détermine dans le diagramme de Hertzsprung-Russell une courbe à droite de laquelle aucun modèle d'équilibre ne peut exister. La position de cette courbe qui est presque verticale dépend quelque peu de la composition chimique et glisse légèrement vers la gauche quand la masse augmente mais au total ces déplacements latéraux ne couvrent guère au plus que quelques centaines de degrés en température effective.

Hayashi n'hésita pas à tirer les conséquences logiques (30) qui découlaient de l'existence de cette zone interdite et qui impliquaient notamment le rejet des chemins évolutifs correspondant à la contraction gravifique de modèles radiatifs (31) qui traversaient justement cette région et qui avaient été acceptés jusque là. En réalité, toute configuration momentanément dans cette partie doit évoluer très rapidement vers un point de la courbe $E=45.5$ où elle peut se stabiliser en réajustant sa structure à celle d'un modèle entièrement convectif. Elle descend ensuite par contraction gravifique le long de cette courbe, restant complètement convective jusqu'au moment où la diminution de l'opacité dans les régions centrales y favorise l'apparition d'un noyau radiatif qui dévie le chemin d'évolution vers des luminosités et des températures effectives correspondant à des valeurs de $E$ inférieures à sa valeur critique et le rapproche du chemin d'évolution radiative de Henyey et al. avec lequel il coïncide pratiquement quand les réactions nucléaires commencent à être efficaces (pour illustration, voir p. ex. (32)).

Etant donné l'importance de ces conclusions, elles ont été soumises à une critique aigüe (33) qui n'a pu que les confirmer au moins qualitativement car une certaine marge d'indétermination subsiste par suite notamment des incertitudes de la théorie de la convection (voir p. ex. (14)). Les conséquences en sont multiples et nous forcent 
en particulier à nous pencher sérieusement sur les toutes premières phases de la contraction capables d'amener l'étoile dans la région de hautes luminosités caractérisant l'évolution à la Hayashi. Et d'abord, par suite des hautes luminosités, les phases convectives de contraction gravifique sont parcourues beaucoup plus rapidement que dans le cas radiatif, surtout pour les faibles masses, ce qui a des incidences considérables sur l'interprétation des amas très jeunes tels que NGC 2264 et sur les quantités d'éléments légers tels que le lithium et le bérylium qui peuvent subsister à la fin de ces phases de contraction convective au cours desquelles ils ont été brassés à grande profondeur jusqu'à des températures élevées.

L'évolution extrèmement rapide qui amène les étoiles sur la séquence convective de contraction gravifique n'a guère fait l'objet encore que d'investigations très approchées (34) mais elle doit être déterminée essentiellement par les processus de refroidissement disponibles aux très basses densités et températures des condensations interstellaires $(35)$ et par les instabilités dynamiques $(34,36)$ correspondant à l'ionisation initiale de l'hydrogène et de l'hélium et à la dissociation de la molécule $\mathrm{H}_{2}$.

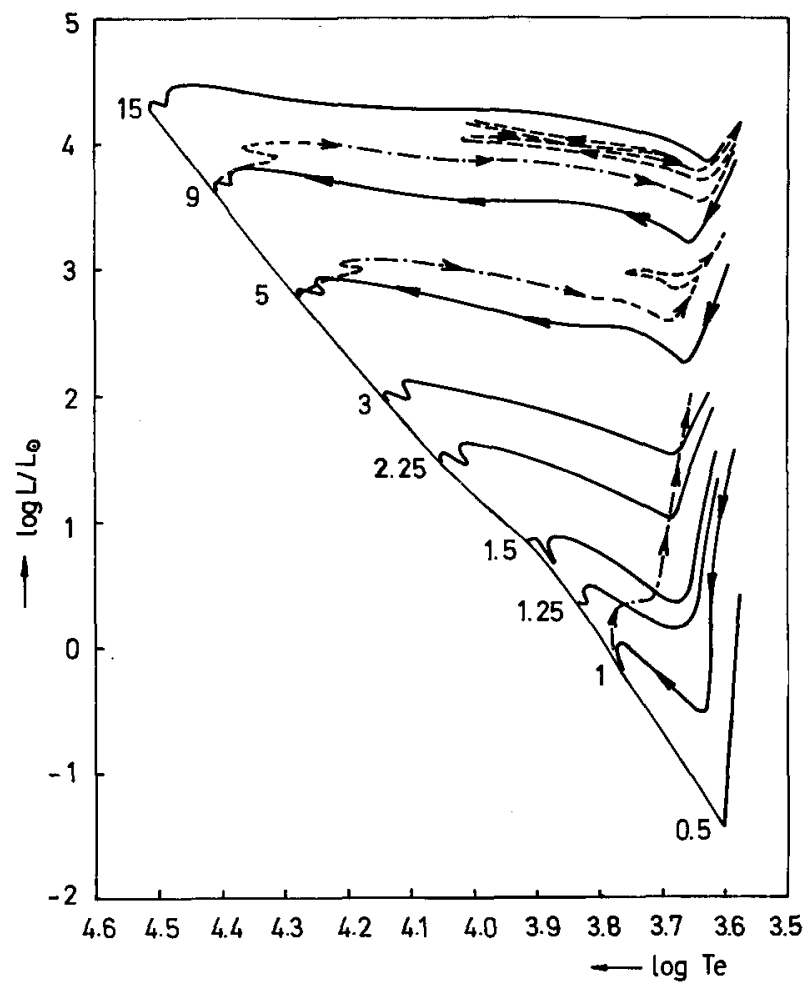

Fig. 4. Evolution schématique dans le diagramme de Hertzsprung-Russell pour quelques valeurs de la masse. Les phases de contraction sont représentées par les courbes en trait plein, les phases postérieures au rebroussement sur la séquence principale, en trait d'axe. 
Pourtant comme Schatzman (37) le fait remarquer, cette instabilité pourrait être réduite fortement ou même supprimée si la condensation initiale était le siège d'une turbulence violente telle que la pression de turbulence cesse d'être négligeable vis-à-vis de la pression gazeuse.

D'autre part, jusqu'ici, on n'a guère fait que soulever la question de l'influence possible d'une rotation ou de champs magnétiques originels qui peuvent sans doute jouer un rôle important à la fois dans la détermination des chemins évolutifs et dans la distribution finale du moment angulaire ou du flux magnétique.

Il est cependant fort douteux que ces facteurs puissent modifier appréciablement les limites de la zone interdite à la droite du diagramme de Hertzsprung-Russell. Ainsi, en pratique, aucun point représentatif d'étoiles ne devrait être observé, dans cette région, qu'il s'agisse d'étoiles fortement évoluées atteignant le sommet de la branche des géantes rouges, car leur évolution vers la droite est limitée par la même courbe critique, ou d'étoiles dans les premiers stades de la contraction gravifique car les phases d'évolution dynamique à droite de la limite doivent être si rapides que les chances de les observer sont extrêmement faibles (voir Figure 4).

Naturellement ceci entraîne la possibilité d'un test précis et quelque peu redoutable de la théorie. Jusque très récemment, les meilleurs calculs, résumés par Auman et Bodenheimer (38), situaient la limite extrême de l'extension des modèles vers les basses températures effectives à des valeurs de l'ordre de 3100 à $3200^{\circ} \mathrm{K}$. Bien qu'en gros l'accord avec les branches des géantes soit remarquable $(\mathbf{2 9}, \mathbf{3 9})$, il existe cependant des géantes rouges possédant des températures effectives nettement inférieures de l'ordre, p. ex., de 2200 à $2500^{\circ} \mathrm{K}$ dans le cas de $o$ Ceti. De plus, dans le cas des étoiles infrarouges observées ces dernières années (40), les estimations de température effective sont tombées encore bien plus bas, aux environs de 800 à $1000^{\circ} \mathrm{K}$ quoique Johnson (41) considère que des corrections de rougissement capables de ramener la température beaucoup plus haut, vers $3000^{\circ} \mathrm{K}$, ne sont pas exclues.

D'autre part, le spectre de ces étoiles froides révèle dans plusieurs cas des bandes d'absorption moléculaires intenses dues à $\mathrm{H}_{2}$, et Auman et Bodenheimer (38) ont cherché à évaluer avec précision les effets de l'opacité due à la vapeur d'eau. Mais l'abaissement correspondant de la limite des températures effectives reste très faible de l'ordre de $100^{\circ} \mathrm{K}$ si les autres paramètres plus ou moins indéterminés dans la théorie de la convection prennent les valeurs habituelles. Toutefois toute modification de ces paramètres qui tend à réduire l'efficacité de la convection dans la zone de transition et à pousser les modèles vers les basses températures voit ses effets largement amplifiés par l'opacité de $\mathbf{H}_{2}$ qui grandit très rapidement quand la température diminue. Auman et Bodenheimer trouvent ainsi qu'une réduction de $l_{t} / H$ par un faible facteur de l'ordre de 0.7 combinée à une augmentation des effets de la friction turbulente et de la dissipation par conduction radiative peuvent, en présence de vapeur d'eau, abaisser la température effective limite aux environs de $2600^{\circ} \mathrm{K}$. Toutefois, il s'agit là d'une limite extrême pour des modèles entièrement homogènes et 
convectifs dont les températures centrales ne dépassent pas $2 \cdot 2 \times 10^{5}{ }^{\circ} \mathrm{K}$ et il n'est pas sûr qu'une étoile issue de la séquence principale puisse jamais l'atteindre. Ainsi, la question de l'accord entre la théorie et l'observation reste malgré tout assez critique et, s'il s'avérait que les étoiles infrarouges ont des températures nettement inférieures à $2000^{\circ} \mathrm{K}$, la théorie serait confrontée avec un problème bien ardu.

Jusqu'ici, nous n'avons considéré que la question des modèles en équilibre hydrostatique ou quasi-statique et nous n'avons rien dit de l'influence des conditions aux limites et des zones d'ionisation de l'hydrogène et de l'hélium sur la stabilité globale des étoiles si ce n'est de rappeler l'instabilité dynamique qui caractérise les toutes premières phases de contraction d'une proto-étoile et l'instabilité vibrationnelle qui affecte les modèles entièrement convectifs d'étoiles de masses très faibles $\left(M \leqslant 0 \cdot 27 M_{\odot}\right)$. Mais cette même instabilité vibrationnelle est d'une importance fondamentale dans l'interprétation des étoiles variables intrinsèques et, là aussi, il semble bien établi à présent que les zones extérieures, sièges de l'ionisation de l'hydrogène et de l'hélium, jouent un rôle capital. C'est Eddington (42) qui attira l'attention sur ce point dans les deux derniers articles qu'il consacra, en 1941-42, aux céphéides, ces mêmes étoiles qui avaient amorcé son intérêt dans le problème de la structure stellaire un quart de siècle plus tôt. Son argument principal reposait sur l'accroissement considérable de la capacité calorifique de ces couches par suite de l'ionisation en cours de l'hydrogène ce qui permettait d'y accumuler de l'énergie à hautes températures, durant la compression et de la libérer à températures plus basses, lors de l'expansion. Elles constituaient ainsi une véritable machine thermique capable de fournir de l'énergie mécanique à l'oscillation compensant l'énergie dissipée par la conduction radiative. Toutefois, il apparut dans la suite qu'en l'absence d'un apport supplémentaire d'énergie dans les couches profondes (43), ce mécanisme était insuffisant et Zhevakin (44), entretemps, avait eu le grand mérite d'attirer l'attention sur l'importance, dans ce cas, des ionisations de l'hélium, particulièrement de la seconde, et des effets d'opacité. Il put montrer que ces facteurs suffisaient pour provoquer une instabilité vibrationnelle appréciable pourvu que le transfert radiatif prédomine encore dans ces régions malgré la convection qui résulte des basses valeurs des $\Gamma$. Dans la suite, la prise en considération de coefficients d'opacité précis et de modèles détaillés des couches extérieures renforça encore cette source d'instabilité et les discussions détaillées de Baker et Kippenhahn (45) de J.P. Cox (46) et de Hoffmeister (47) confirmèrent définitivement l'effet global déstabilisant des couches extérieures dans les céphéides quoique les rôles respectifs des différentes zones d'ionisation et des effets de capacité calorifique ou d'opacité soient souvent si intimement mêlés qu'il n'est pas toujours très aisé de les distinguer. Ceci est vrai également de la question délicate des déphasages typiques entre les variations de luminosité et de rayon (46).

Enfin, les remarquables résultats de Christy (48) déduits de l'intégration directe des équations non-linéaires du problème, confirmés dans certains aspects par les investigations de J.P. Cox et A. N. Cox (49) et de leurs collaborateurs ainsi que par Alyoshin 
(50) montrent que ce type d'instabilité peut effectivement conduire, sous l'action des effets non-linéaires, à des oscillations finies dont les amplitudes et l'anharmonicité sont en bon accord avec l'observation.

De plus, le mécanisme déstabilisant ne semble effectif que dans une mince bande du diagramme de Hertzsprung-Russell en accord satisfaisant avec la localisation des céphéides et partant fournit également une explication réelle de la relation PériodeLuminosité.

D'autre part, ces résultats combinés à la théorie de l'évolution qui montre qu'une même étoile peut traverser plusieurs fois dans un sens et dans l'autre la bande d'instabilité, ouvrent d'intéressantes possibilités d'interpréter les variations de périodes parfois observées et suggèrent toute une série de tests statistiques.

La plus grande faiblesse de la théorie tant du point de vue linéaire que non-linéaire réside, comme ses protagonistes eux-mêmes l'ont souvent remarqué, dans les difficultés très sérieuses qui s'opposent à présent à une évaluation réaliste des effets mécaniques et thermiques de la convection dans les couches extérieures au cours de la pulsation. D'autre part, les conditions aux limites à la 'surface' de l'étoile restent assez délicates à formuler d'une façon entièrement satisfaisante en particulier si l'étoile est entourée d'une couronne à température élevée (51). Enfin, le passage du cas linéaire au cas non-linéaire soulève des problèmes mathématiques et physiques difficiles et parfois intriguants. Ainsi, les calculs non-linéaires semblent stabiliser assez rapidement l'oscillation autour d'un des modes linéaires, parfois le mode fondamental, parfois le premier mode alors que l'instabilité linéaire affecte à peu près également ces deux modes et peut même, dans certains cas, s'étendre à des modes plus élevés, tous ces modes ayant d'ailleurs des périodes, en général, strictement incommensurables.

De toute façon, on peut certainement conclure que la prise en considération de conditions de surface plus réalistes et de leurs implications pour les zones de transition quasi-convectives liées à l'ionisation de l'hydrogène et de l'hélium a contribué puissamment, au cours des 10 ou 12 dernières années, à la solution de problèmes relevant typiquement du domaine de la structure interne des étoiles: interprétation de la branche des géantes rouges, modifications profondes de nos idées sur l'évolution par contraction gravifique, existence d'une région interdite dans le diagramme de Hertzsprung-Russell aux températures effectives plus basses que quelque $2500^{\circ} \mathrm{K}$, progrès marquants dans l'interprétation des variables intrinsèques des types céphéides, RR Lyra et peut-être des céphéides naines.

D'autre part, j'ai à peine fait allusion aux interactions plus délicates entre ces zones d'ionisations quasi-convectives et l'atmosphère qui, p. ex. dans le soleil, sont sans doute responsables de la grande variété de phénomènes transitoires ou semi-périodiques observés à sa surface aussi bien que de l'échauffement des couches chromosphériques et de la couronne. Tout en m'écartant du sujet même de cette conférence et en l'allongeant indûment, la discussion de ces différents aspects m'eût aussi rapide- 
ment entraîné dans un domaine qui sort largement de ma compétence. Cependant, la découverte par Leighton $(52)$ de la nette périodicité $(\simeq 300 \mathrm{sec})$ sur quelques cycles successifs de champs de vitesses à petites échelles $(<5000 \mathrm{~km})$ à la surface du soleil a renouvelé l'intérêt (53) dans l'étude des ondes et des oscillations atmosphériques du soleil. La plupart de ces investigations se rapportent soit aux modes propres d'oscillations des couches supérieures convectivement stables ou à la propagation, dans ces couches, d'ondes excitées par la convection turbulente sous-jacente soit par pénétration, soit par effets gravifiques, soit encore par des variations aléatoires des conditions aux limites à la surface de séparation entre les deux zones. Maintenant que les familles possibles de modes d'oscillations non-radiales dans un milieu contenant une zone superadiabatique sont mieux connues (54), il me paraîtrait intéressant de réétudier le problème global des oscillations des couches extérieures du soleil couvrant à la fois la zone atmosphérique stable et la zone d'ionisation de l'hydrogène vers le sommet de laquelle, malgré l'établissement de la convection, de forts gradients superadiabatiques peuvent subsister.

Dans le cas des champs périodiques de vitesse de Leighton, étant donné la rapidité des oscillations observées, il s'agit vraisemblablement de modes acoustiques ou modes-p mais leur excitation pourrait être liée à l'existence de ces gradients superadiabatiques (55) et les possibilités de couplage avec les modes de gravité, ou modes-g, stables et instables devraient être prises en considération.

Notons aussi qu'en présence de rotation ou de champs magnétiques, certains des modes-g instables et sans doute particulièrement ceux de grandes longueurs d'ondes horizontales, les autres se confondant avec la convection turbulente proprement dite, peuvent devenir 'overstable' $(\mathbf{5 6})$ et acquérir des fréquences réelles $(\mathbf{5 7}, \mathbf{5 8})$ liées à la vitesse angulaire de rotation ou à l'intensité du champ magnétique. Les périodes correspondantes seraient vraisemblablement très longues mais elles pourraient être significatives pour d'autres aspects des phénomènes solaires.

Poussant les choses plus loin, on peut se demander si, à part la convection profonde ordinaire, toute possibilité de connexion directe entre l'atmosphère superficielle et les couches beaucoup plus profondes est totalement exclue. D'habitude, du moins dans les modèles possédant des zones radiatives intermédiaires étendues, on considère que l'intérieur profond n'influence guère l'atmosphère que par le champ gravifique qu'il y établit et par la quantité totale d'énergie qu'il dégage. Celle-ci, sous forme de radiation, ne progresse d'ailleurs que très lentement vers la surface et en prenant dans chaque couche des propriétés d'équilibre qui effacent totalement les caractéristiques des couches plus profondes. Mais, p. ex., la théorie des taches solaires et de leur cycle, proposée par Alfvén (59) il y a quelques années ainsi que la version de Walén (60) faisaient appel à une telle interaction profonde liée à la propagation jusqu'à la photosphère d'ondes hydromagnétiques nées à la surface du noyau convectif supposé, à l'époque, exister dans les régions centrales du soleil. La diminution de densité accompagnant la concentration du champ magnétique dans un tube ou dans un globule 
stabilisé par ce champ même et en équilibre de pression totale avec le milieu ambiant fait apparaître, comme l'ont signalé Parker (61) et Jensen (62), une force d'Archimède qui pousse ce globule ou ce tube vers la surface. Récemment, cette possibilité a été discutée (63) du point de vue du transport des propriétés de composition chimique profonde vers la surface.

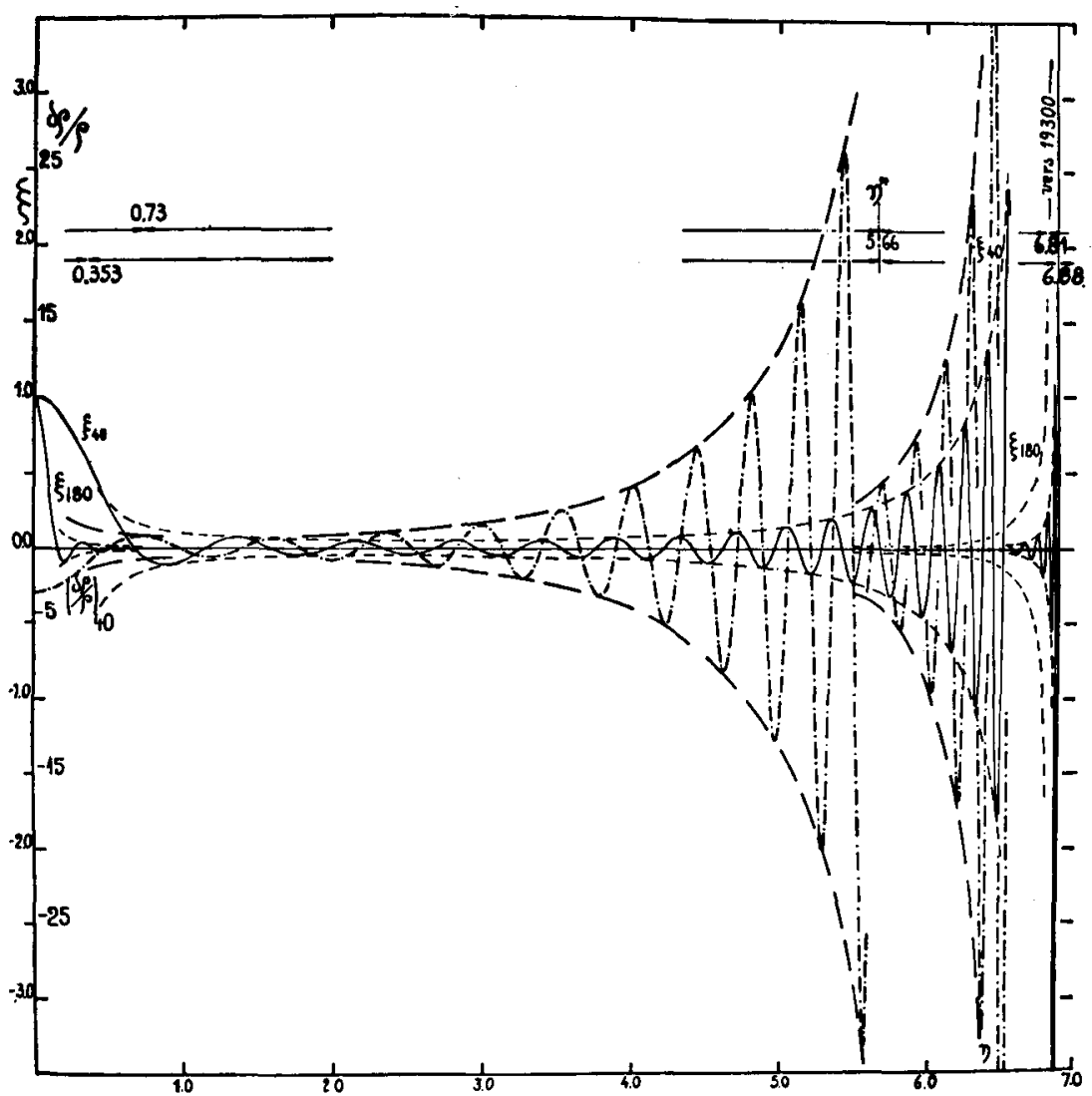

FIG. 5. Comportement de l'amplitude relative $\xi=\delta r / \boldsymbol{r}$ (trait plein) et de $\delta \rho / \rho$ (trait pointillé) pour des modes élevés de pulsation radiale.

Le comportement de l'amplitude des modes très élevés d'oscillations radiales possède un aspect assez curieux (64) qui suggère également quelque possibilité d'interaction. En effet, cette amplitude commence par décroître très rapidement à partir du centre pour prendre des valeurs très faibles partout sauf près de la surface où elle peut atteindre des valeurs extrêmement grandes. Dans la région intermédiaire étendue, l'amplitude est si petite que la dissipation radiative ou visqueuse doit être très faible 
et ainsi il se peut que la plus grande partie de l'énergie gagnée par l'onde très près du centre par suite des réactions nucléaires ne soit dissipée qu'aux environs de la surface. Bien que nous ne disposions pas, dans le cas des oscillations non-radiales, d'une théorie asymptotique adéquate, on peut néanmoins s'attendre à un comportement quelque peu analogue au moins pour les modes-p. Dans ce cas, quels peuvent être, p. ex., les effets de ces modes-p certainement excités dans les noyaux convectifs de toute une classe d'étoiles?

Enfin, au cours des 10 à 15 dernières années, une autre notion nouvelle, celle de 'vent solaire' ou plus généralement de 'vent stellaire' est venue préciser le comportement des couches les plus extérieures des étoiles. Suggérée d'abord par Biermann (65) sur la base d'observations cométaires, cette notion s'est vue, sur cette courte période, dotée d'un statut théorique satisfaisant à beaucoup de points de vue grâce surtout aux travaux de Parker (66), puis définitivement confirmée, dans le cas du soleil, par les mesures effectuées à bord des satellites artificiels. Tout récemment, les fluctuations très rapides $(<1 \mathrm{sec})$ dans le flux des sources-radio de très petits diamètres angulaires (67) y ont révélé une structure surprenante caractérisée par des irrégularités de très petites dimensions $(\simeq 200 \mathrm{~km})$ qui en constitue peut-être une autre caractéristique significative.

$\mathrm{Si}$, dans le cas du soleil, la perte de masse correspondante est si faible qu'on puisse la rejeter immédiatement en tant que facteur susceptible d'influencer sa structure interne ou son évolution, il n'en est sans doute pas de même pour d'autres étoiles notamment les supergéantes (68) et peut-être même les géantes rouges qui ont longuement retenu notre attention ici. A ce point de vue, la théorie des vents stellaires ne sera vraiment satisfaisante qu'au moment où elle couvrira adéquatement son origine aussi bien que sa description cinématique et reliera son intensité aux autres propriétés de l'étoile. Il s'agit là, certainement d'un problème très difficile lié à la théorie détaillée de l'évaporation compte tenu de l'anisotropie des fonctions de distribution dans les couches très extérieures et aux conséquences, au moins dans certains cas, de l'absorption sélective d'ions accélérés et de l'activité acoustique engendrée par la convection turbulente dans les couches sub-photosphériques.

Quoiqu'il en soit, tous ces derniers points que nous n'avons pu qu'effleurer très superficiellement suggèrent une foule de problèmes dont la solution, sans aucun doute, tissera des liens de plus en plus étroits entre les questions de structure interne et le comportement des couches les plus extérieures, nous livrant enfin une vue globale de l'étoile y compris ses interactions avec le mileu interstellaire dans lequel elle baigne.

\section{Bibliographie générale}

Hayashi, C. (1966) A. Rev. Astron. Astrophys., 4, 171.

Hayashi, C., Hashi, R., Sugimoto, D. (1962) Evolution of the Stars, Progr. Theor. Phys. Suppl., 22. Pecker, J.-C. (1960) 9e Coll, Intern. Astrophys. Liège 1959, Mém. Soc. Roy. Sci. Liège, Se sér., $3,343$. 


\section{Références}

1, Voir p. ex.: Odgers, G.J. (1957) Publ. Dom. Astrophys. Obs., Victoria, 10, 393.

2. Voir en particulier Fowler, R.H. (1930) Mon. Not. R. astr. Soc., 91, 63. - Strömgren, B., ibid., 466. - Cowling, T. G., ibid., 472. - Hopf, E., ibid., 653.

3. Milne, E.A. (1930) Mon. Not. R. astr. Soc., 91, 4; (1932) ibid., 92, 610; (1945) ibid., 105, 146; (1931) Z. Astrophys., 3, 253.

4. Russell, H.N. (1931) Mon. Not. R. astr. Soc., 91, 952. - Rosseland, S. (1932) Z. Astrophys., 4, 255. - Cowling, T. G. (1932) Z. Astrophys., 4, 331.

5. Unsöld, A. (1930) Z. Astrophys., 1, 138.

6. Chandrasekhar, S., Münch, G. (1946) Astrophys. J., 104, 446. - Unsöld, A. (1955) Physik der Sternatmosphären, 2ème éd., Springer-Verlag, Berlin.

7. Bierman, L. (1937) Astronom. N., 264, 361 et 395.

8. Schwarzschild, M. (1958) Structure and Evolution of the Stars, Princeton Univ. Press, § 11.

9. Ezer, D., Stein, R.F., Cameron, A.G.W. (1963) Astron. J., 68, 278. - Hofmeister, E., Weigert, A. (1964) Z. Astrophys., 59, 119.

10. Faulkner, J., Griffiths, K., Hoyle, F. (1964) Mon. Not. R. astr. Soc., 129, 363. Voir aussi: Ruben, G.V. (1963) Astr. Zu., 40, 855.

11. Ledoux, P., Schwarzschild, M., Spiegel, E. (1961) Astrophys. J., 133, 184.

12. Böhm, K.H., Richter, E. (1959) $\quad$ Z. Astrophys., 48, 231.-Böhm, K.H.(1963) Astrophys. $J ., 137,881$. - Spiegel, E. (1964) Astrophys. J., 139, 959.

13. Cox, A.N. (1965) Stars and Stellar Systems, 8, Ed. by L.H. Aller and D.B. McLaughlin, Univ. of Chicago Press, Chicago, p. 195; (1964) J. Quantit. Spectrosc. Radiat. Transfer, 4, 737. - Cox, A.N., Stewart, J.H., Eilers, D.D. (1965) Astrophys. J. Suppl. 11, 1. - Cox, A. N., Stewart, J.H., ibid., 22. - Mayer, H.L. (1964) J. Quantit. Spectrosc. Radiat. Transfer, 4, 585.

14. Voir p. ex.: Henyey, L.G., Vardya, M.S., Bodenheimer, P. (1965) Astrophys. J., 142, 841.

15. Sack, N. (1966) Ann. Astrophys., 29, 633.

16. Vitense, E. (1953) Z. Astrophys., 32, 135.

17. Böhm-Vitense, E. (1958) Z. Astrophys., 46, 108.

18. Voir p. ex.: Baker, N.H., Temesvary, S. (1966) Tables of Convective Stellar Envelope Models, Inst. Space Studies, NASA, New York.

19. Schatzman, E. (1962) Ann. Astrophys., 25, 18.

20. Osterbrock, D.E. (1953) Astrophys. J., 118, 529.

21. Limber, D. N. (1958) Astrophys. J., 127, 387.

22. Hayashi, C., Nakano, T. (1963) Progr. Theor. Phys., 30, 460.

23. Gabriel, M. (1966) Coll. on Late-Type Stars, Trieste, p. 347.

24. Gamow, G., Keller, G. (1945) Rev. Mod. Phys., 17, 125. - Hayashi, C. (1947) Progr. Theor. Phys., 2, 127. - Hayashi, C. (1949) Phys. Rev., 75, 1619. - Gardiner, J.G. (1951) Mon. Not. R. astr. Soc., 111, 102. - Dumézil-Curien, P. (1954) Ann. Astrophys., 17, 197.

25. Sandage, A.R., Schwarzschild, M. (1952) Astrophys. J., 116, 463.

26. Schwarzschild, M., Rabinowitz, I., Härm, R. (1953) 118, 326.

27. Hoyle, F., Schwarzschild, M. (1955) Astrophys. J. Sup. $2,1$.

28. Haselgrove, C. B., Hoyle, F. (1956) Mon. Not. R. astr. Soc., 116, 527; (1958) ibid., 118, 519 ; (1959) ibid., 119, 112. - Kippenhahn, R., Temesvary, S., Biermann, L. (1958) Z. Astrophys., 46, 257. - Simoda, M., Obi, S. (1958) Publ. Astr. Soc. Japan, 10, 26. - Simoda, M. (1961) Publ. Astr. Soc. Japan, 13, 424. - Schwarzschild, M., Selberg, H. (1962) Astrophys. J., 136, 150. - Swamy Krishna, K.S., Kushwaha, R.S. (1962) Astrophys. J., 135, 802. - Iben, I., Jr. (1967) Astrophys. J., 147, 624.

29. Hayashi, C., Hoshi, R. (1961) Publ. Astr. Soc. Japan, 13, 442.

30. Hayashi, C. (1961) Publ. Astr. Soc. Japan, 13, 450.

31. Henyey, L.G., Le Levier, R., Levée, R.D. (1955) Publ. Astr. Soc. Pacific, 67, 154. Cf. also Brownlee, R.R., Cox, A. N. (1961) Sky and Telescope, 21, 252.

32. Hayashi, M. (1965) Publ. Astr. Soc. Japan, 17, 177. - Ezer, D. (1966) Coll. on Late-Type Stars, Trieste, p. 357. 
33. Faulkner, F., Griffiths, K., Hoyle, F. (1963)

Mon. Not. R. astr. Soc., 126, 1 ; (1965) ibid., 129, 363. - Ezer, D., Cameron, A.G.W. (1963)

Icarus, 1, 422; (1965) Canadian J. Phys., 43, 1497. - Weymann, R., Moore, E. (1963) Astrophys. J., 137, 522.

34. Cameron, A.G.W. (1963) Icarus, 1, 13. - Hayashi, C., Nakano, T. (1965) Progr. Theor. Phys., 34, 754. - Upton, E.R.L., Little, S.J., Dworetsky, M.M. (1967) Astrophys. J. (sous presse); cf. also ibid., (1967) Astron. J., 72, 308.

35. Gaustad, J.E.(1963) Astrophys.J., 138, 1050. - Gould, R.J.(1964) Astrophys. J., 140, 638.

36. Ledoux, P. (1958) Hdb. Physik, Ed. by S. Flügge, Springer-Verlag, Berlin, t. LI, p. 660.

37. Schatzman E. (1967) Ann. Astrophys. 30, 963.

38. Auman, J. R., Bodenheimer. (1967) Astrophys. J., 149, 641.

39. Barbaro, G., Dallaporta, N., Nobili, L. (1966) Coll. on Late-Type Stars, Trieste, p. 368.

40. Neugebauer, G., Martz, D. E., Leighton, R.B. (1965) Astrophys. J., 142, 399. - Johnson, H. L., Mendoza, E.E., Wisniewski, W.Z. (1965) Astrophys. J., 142, 1249. - Johnson, H.L. (1966) A. Rev. Astron. Astrophys., 4, 193.

41. Johnson, H.L. (1966) 121st meeting A.A.S., Hampton, Va, March.

42. Eddington, A.S. (1941) Mon. Not. R. astr. Soc., 101, 182; (1942) ibid., 102, 154.

43. Cox, J.P. (1955) Astrophys. J., 122, 286.

44. Zhevakin, C.A. (1952) Astr. Zu., 29, 37; (1954) ibid., 31, 335; (1955) ibid., 32, 124 etc. Cf. A. Rev. Astron. Astrophys., 1, $1963,367$.

45. Baker, N., Kippenhahn, R. (1962) Z. Astrophys., 54, 114; (1965) Astrophys. J., 142, 868.

46. Cox, J.P. (1959) Astrophys. J., 130, 296; (1963) ibid., 138, 487.

47. Hofmeister, E. (1965) Thesis (München); (1965) Mitt. Astr. Ges., 19, 90; (1965) Proc. I.A.U. Coll., Bamberg, p. 224 (Kl. Veröff. Remeis-Ternw. Bamberg, 4, no. 40).

48. Christy, R.F. (1966) Astrophys. J., 144, 108; (1966) A. Rev. Astron. Astrophys. 4, 353; (1967) I.A.U. Symp. no. 28; Aerodynamic Phenomena in Stellar Atmospheres, Nice 1965, p. 105.

49. Cox, J.P., Cox, A.N., Olsen, K.H., King, D.S., Eilers, D.D. (1966) Astrophys. J., 144, 1038. - King, D.S., Cox, J.P., Eilers, D.D. (1966) Astrophys. J., 144, 1069.

50. Alyoshin, V.I. (1964) Astr. Zu., 41, 201; (1966) ibid. (sous presse).

51. Voir p. ex.: Unno, W. (1965) Publ. Astr. Soc. Japan, 17, 205. - Simon, R. (1964) Astrophys. Norv., 9, 113.

52. Leighton, R.B. (1961) Nuovo Cimento, Suppl. 22, 321. - Leighton, R.B., Noyes, R.W., Simon, G.W. (1962) Astrophys. J., 135, 474. - Evans, J., Michard, R. (1962) Astrophys. J., 136, 493. - Leighton, R. B. (1963) A. Rev. Astron. Astrophys., 1, 13.

53. Whitney, C.A. (1958) Smithsonian Contr. Astrophys., 2, 365; (1963) Astrophys. J., 138, 537. Kahn, F.D. (1961) Astrophys. J., 134, 343; (1962) ibid., 135, 547. - Schmidt, H.V., Zirker, J.B. (1963) Astrophys.J., 138,1310. - Bahng, J., Schwarzschild, M. (1963) Astrophys. J., 137, 901.. - Schatzman, E. (1964) Astrophys. Norv., 9, 283. - Moore, D.W., Spiegel, E. A. (1964) Astrophys. J., 139, 48. - Orrall, F.Q. (1965) Astrophys. J., 141, 1131. - Uchida, Y. (1965) Astrophys. J., 142, 335. - Souffrin, P. (1966) Ann. Astrophys., 29, 55. - Kato, S. (1966) Astrophys. J., 143, 893; ibid., 144, 326.

54. Ledoux, P., Smeyers, P. (1966) C.R. Acad. Sci., Paris, 262, 841. - Smeyers, P. (1966) Ann. Astrophys., 29, 539.

55. Spiegel, E. (1964) Astrophys. J., 139, 959.

56. Chandrasekhar, S. (1961) Hydrodynamic and Hydromagnetic Stability. Clarendon Press, Oxford, Chaps. III and IV.

57. Voir p. ex.: Ledoux, P. (1951) Astrophys. J., 114, 373.- Ledoux, P., Simon, R. (1957) Ann. Astrophys., 20, 185. - Ledoux, P. (1967), The Magnetic and Related Stars. AAS-NASA Symposium, Greenbelt (U.S.A.), 1965, Ed. by R.C. Cameron, Mono Book Corporation, Baltimore, p. 65.

58. Cretin, M., Tassoul, J. L. (1965) Ann. Astrophys., 28, 982.

59. Alfvén, H. (1945) Mon. Not. R. astron. Soc., 105, 3 and 382.

60. Walén, C. (1944) Ark. Nat. Astr. Fys., 30A, no. 15; (1949) On the Vibratory Rotation of the Sun. Henrik Lindstähls Bokhandel, Stockholm. 
61. Parker, E. N. (1955) Astrophys. J., 121, 491.

62. Jensen, E. (1955) Ann. Astrophys., 18, 127; (1957) Astrophys. Norv., 5, 289.

63. Gurm, H.S., Wentzel, D.G. (1967) Astrophys. J., 149, 139.

64. Ledoux, P. (1963) Bull. Acad. R. Belgique, Cl. Sci., 5e série, 49, 286.

65. Biermann, L. (1951) Z. Astrophys., 29, 274; (1957) Observatory, 77, 109.

66. Parker, E.N. (1958) Astrophys. J., 128, 664;(1960) ibid. 132, 821; (1964) ibid., 139, 72, 93 and 690; (1965) ibid., 141, 322.

67. Hewish A., Scott, F.P., Wills, D. (1964) Nature, 203, 1214. - Little, L.T., Hewish, A. (1966) Mon. Not. R. astron. Soc., 134, 221. - Salpeter, E. E. (1967) Astrophys. J., 147, 433.

68. Deutsch, A.J. (1956) Astrophys. J., 123, 210. Voir aussi: Weymann, R. (1963) A. Rev. Astron. Astrophys., 1, 97. 